\title{
Improving the dynamic range in distributed anti-Stokes Raman thermometry by means of susceptibility asymmetry
}

\author{
Lívia A. Ribeiro, João B. Rosolem, Antônio O. Toledo \\ Instituto Nacional de Pesquisas Espaciais - INPE \\ Centro de Pesquisa e Desenvolvimento em Telecomunicações - CPqD \\ Instituto de Estudos Avançados - IEAv
}

\begin{abstract}
In this paper it is verified, from experimental results, that the Raman susceptibility asymmetry is found as a key factor to improve dynamic range in distributed anti-Stokes Raman thermometry sensor which operates in stimulated regime. Improvements are discussed from experimental results of the spatial evolution of the anti-Stokes and Stokes branches for different Raman anti-Stokes wavelengths.
\end{abstract}

Stimulated Raman scattering, anti-Stokes Raman scattering, Raman susceptibility, Distributed sensor, Standard optical fiber, OTDR.

\section{INTRODUCTION}

Distributed fiber sensors are the state of the art for sensing huge structures as pipelines, aircraft, boreholes and many other application, since it can measure the temperature at any point of the whole extension with finite precision. Since the first distributed sensor was developed ${ }^{1,2}$ in 1985 many approaches has been discussed to manage the challenges in using Raman scattering as sensor mechanism ${ }^{3}$. It has also been done some efforts in order to increase spatial resolution in small sensors as well as to increase the sensing range at the same time that sensor's accuracy is improved ${ }^{4}$ at long distance applications. Most of the distributed Raman sensors works on spontaneous regime, where the input power required is very low. Nevertheless, when distributed sensors work on stimulated regime the spatial evolution of the Stokes-anti-Stokes coupling may be a key to improve receiver's performance, depending on the strength of the coupling and the incident electromagnetic field. In this paper we discuss, from experimental results, those points and their crucial importance to building a sensor based on distributed anti-Stokes ratio thermometry (DART) operating at stimulated regime, we also measure the anti-Stokes wavelength dependence of this factor for.

\section{BACKGROUND}

Raman scattering occurs due to the interaction between light and optical phonons presents in a continuum medium. In solid materials it can result in an electronic or a vibrational transition, depending on the energy of the incident photon. In such interaction, medium can lose energy (anti-Stokes) or acquire energy (Stokes) by means of scattering. As it is known molecular vibrational states - the phonons - obey the Bose-Einstein statistics distribution energy, which is a function of temperature. At room temperature most of the molecules are at the lower energy state, so anti-Stokes scattering is even rarer than Stokes. Since the scattered intensity is known one can determine the medium temperature by determining the anti-Stokes number of photons according to equation (1).

$$
f(T)=\left[\exp \left(\frac{h c v}{K T}\right)-1\right]^{-1}
$$

Stimulated Raman scattering (SRS) can be described by the nonlinear polarization in a classical approach ${ }^{5}$. The physical parameter which describe Raman scattering is the imaginary part of the third order susceptibility which in turn show a frequency shift asymmetry with respect to pump frequency ${ }^{7}$. The spatial evolution of the Raman branches is described by equation (2), when SRS is the predominant effect, due the susceptibility nature, anti-Stokes branch experiences attenuation and Stokes experiences gain. L, S, A in equation (2) meas laser pump, Stokes and anti-Stokes respectively.

21st International Conference on Optical Fiber Sensors, edited by Wojtek J. Bock, Jacques Albert, Xiaoyi Bao, Proc. of SPIE Vol. 7753, 77532W · @ 2011 SPIE · CCC code: 0277-786X/11/\$18 · doi: 10.1117/12.885939 


$$
\begin{aligned}
& \frac{d A_{S}}{d z}=-\gamma_{S} A_{s}+\kappa_{S} A_{a}^{*} e^{i \Delta k z} \\
& \frac{d A_{A}}{d z}=-\gamma_{A} A_{A}+\kappa_{A} A_{S}^{*} e^{i \Delta k z}
\end{aligned} \quad \text {, with } \quad \alpha_{j}=\frac{-3 i \omega_{j}}{n_{j} c} \chi_{R}\left(\omega_{j}\right)\left|A_{L}\right|^{2}
$$

On the other hand, the four-wave mixing term (second term on the right) demonstrate in some situations a gain in antiStokes branch as a result of the Stokes - anti-Stokes coupling ${ }^{6}$. In a situation where wavevector mismatch is small, the four-wave mixing term can be ignored. As it was verified in our case, SRS is predominant leading to Raman anti-Stokes wavelength loss to provoke a dramatical deterioration on dynamical range in distributed temperature sensors operating in stimulated scattering regime.

\section{EXPERIMENTAL APPARATUS}

The measurements were carried out using a $1547 \mathrm{~nm}$ laser pump with $0 \mathrm{dBm}$ modulated externally by pulse generator, set at 200ns. The $1547 \mathrm{~nm}$ pulse beam was amplified in EDFA which allowing average power up to $200 \mathrm{~mW}$. Light was launched in a test fiber $12 \mathrm{~km}$ long maximum by using a circulator, an impedance matching was placed at the far end of the fiber in order to avoid reflection. Three different filters was used to allow passing the wavelengths of interest. The receptor was developed in our laboratory for each Raman branch individually and it consisted of transimpedance amplifier (TIA 1 and TIA 2) followed by two stages of amplification with a total gain of $60 \mathrm{~dB}$ and $200 \mathrm{kHz}$ electrical bandwidth for the Stokes and anti-Stokes branches. Signal was detected using a digital oscilloscope.

\section{RESULTS}

Measurements was carried out using two different transimpedance amplifier (TIA 1 and TIA 2) to compare the optical time domain reflectometry for Stokes (RS-OTDR) and anti-Stokes (RA-OTDR). A set of test measurements was done in order to ensure the correct TIA operation.
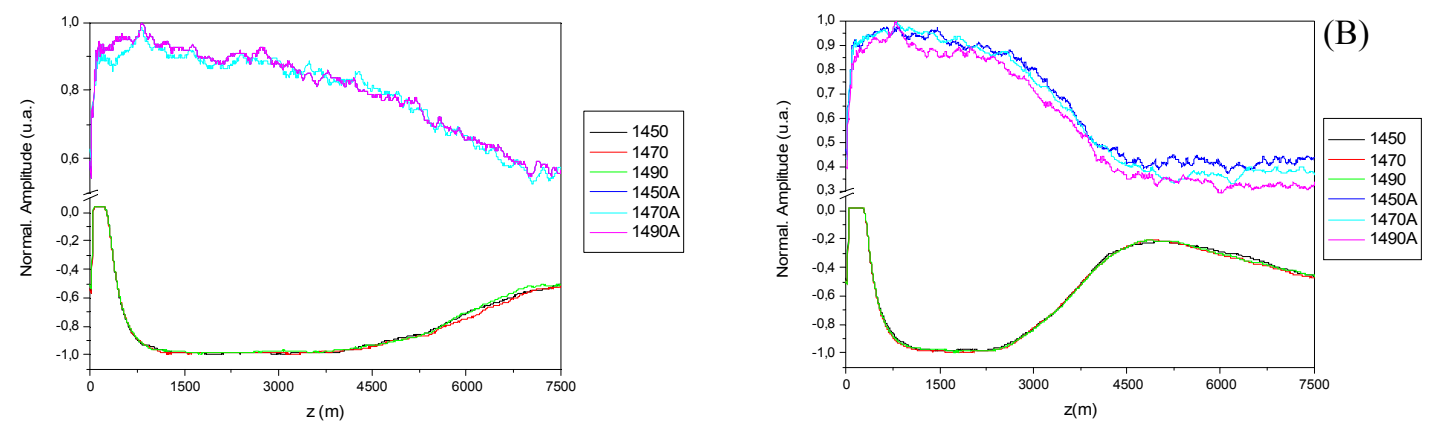

Figure 1: RS- and RA- OTDR curves for three different anti-Stokes wavelengths and two different values of average power input (A) Pin $=60 \mathrm{~mW}$ and (B) $80 \mathrm{~mW}$. Stokes data are kept at the same wavelength $(1650 \mathrm{~nm})$ and it was acquired for each anti-Stokes measurement.

The Raman susceptibility is a function of Raman frequency shift whose its maximum occurs at the resonance frequency ${ }^{5,7}$ so it is expected the Raman loss (or gain) also show some wavelength dependence. In order to quantify this dependence we have measured the RA-OTDR for three wavelengths belonging to anti-Stokes branch, namely $7.1 \mathrm{THz}$ $(1490 \mathrm{~nm}) ; 9.6 \mathrm{THz}(1470 \mathrm{~nm}) ; 12.2 \mathrm{THz}(1450 \mathrm{~nm})$. It was found no measurable wavelength dependence, according to figure 1. From the spatial evolution differential equation and experimental results can be verified that the maximum Raman gain occurs in a position $\left(\mathrm{z}^{*}\right)$ close to the minimum loss point (figure 1). In figure 2, the $\mathrm{z}^{*}$ obtained from experimental data are plotted as a function of power input, for Stokes and anti-Stokes data. Since experimental data are accompanied by uncertainty, the minimum anti-Stokes position are compatible with maximum Stokes position, moreover OTDR-like techniques have a precision length that in this case is about $40 \mathrm{~m}$. 


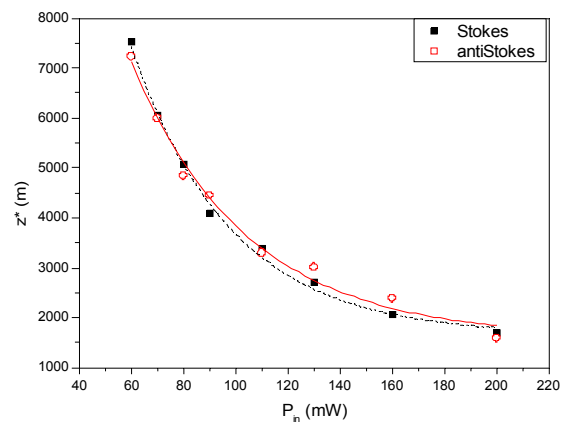

Figure 2: Position of maximum Raman gain (Stokes) and minimum Raman loss (anti-Stokes) position measured from RS- and RAOTDR for several power input.

It was found from figure 2, that the anti-Stokes Raman signal is serious deteriorated from the same point where Stokes signal reach its maximum. In a distributed thermometry sensor based on Raman scattering, the anti-Stokes amplitude is the fundamental parameter to be measured in order to determine the signal-noise ratio, dynamical range and also length precision ${ }^{1}$. In this way, we have performed some experiments to verify the impact of the anti-Stokes depletion in a distributed sensor operating in stimulated regime. Distributed Raman thermometry is presented for two different coil lengths (figure 3), we also take into account the susceptibility wavelength dependence in this case to preform temperature sensibility dependence (figure 4).

(A)

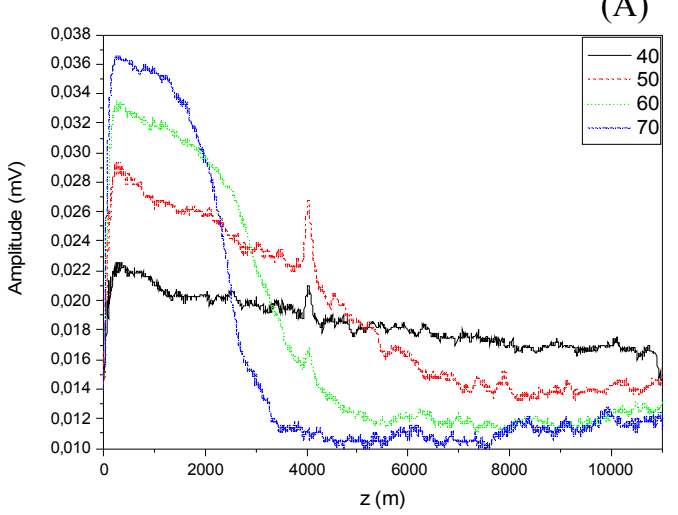

(B)

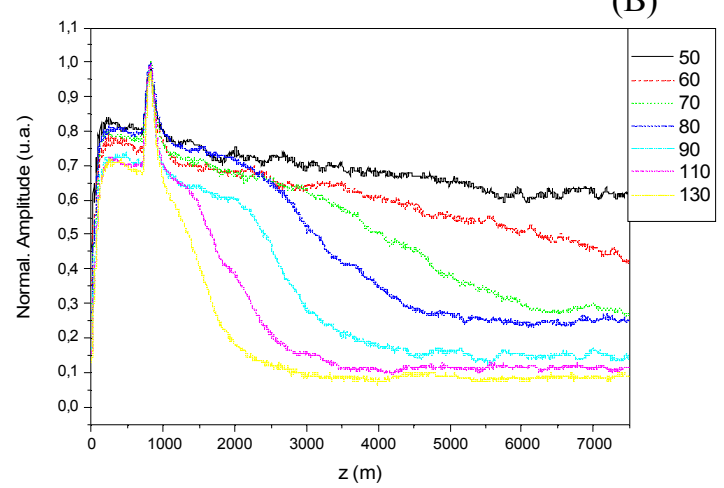

Figure 3: RA-OTDR obtained for two optical fiber coils: (A) $\mathrm{L}=10876 \mathrm{~m}$ and (B) $\mathrm{L}=7650 \mathrm{~m}$. Figure (B) is normalized for comparing, since there are a wide range of average input power involved. Anti-Stokes wavelength was taken at $1450 \mathrm{~nm}(12,2 \mathrm{THz})$.

A small portion of fiber $100 \mathrm{~m}$ long, placed at different point of the fiber link, was heated to $80^{\circ} \mathrm{C}$. This $100 \mathrm{~m}$ fiber works as a marker which allow one to verify the dynamical range length as function of power input. First, the small coil was placed at the middle of the link (figure 3A), at this length average power input is limited to $60 \mathrm{~mW}$, whilst if the coil is placed at the beginning of the link average power can be increased up to $130 \mathrm{~mW}$ before the RA-OTDR starts to deteriorate, according to figure 3B. Unlike the spontaneous regime, where dynamical range scales linearly with power input, at the stimulated regime it goes with an exponential decay whose the argument is the Raman loss coefficient related with the Stokes gain coefficient causing some damage effects in a DART signal. According to the Raman gain profile, most of the scattering events is found at $13 \mathrm{THz}$ frequency shift, so this is the same parameter used to predict maximum Raman amplification as the Stokes pump propagates down the fiber in stimulated regime (equation 2), as a consequence the silica Raman frequency shift is also responsible to generate the maximum anti-Stokes attenuation due to the asymmetry on Raman susceptibility. Therefore, in a SRS distributed sensor one should find an intermediate point to acquire a good signal-noise ratio.

The wavelength susceptibility dependence was verified by measurements of RA-OTDR for three anti-Stokes wavelengths in an optical fiber link heated up to $80^{\circ} \mathrm{C}$, average input pump power were $50 \mathrm{~mW}, 60 \mathrm{~mW}$ and $70 \mathrm{~mW}$. The 
heated portion of the fiber was placed at the first kilometer of the link, so no signal deterioration was observed at that point for those power input, figure (4) presents some of this curves.
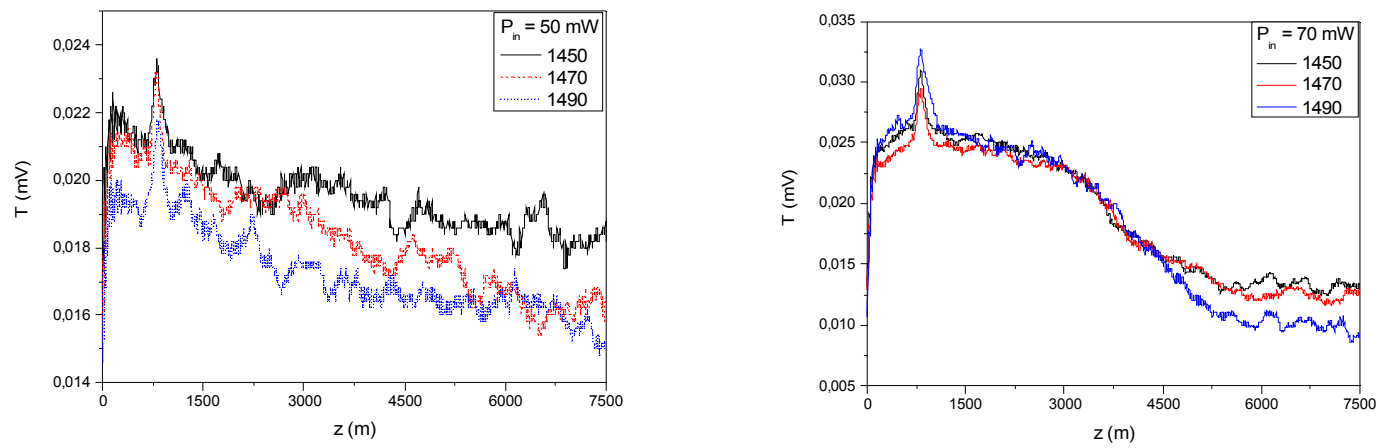

Figure 4. RA-OTDR obtained three anti-Stokes wavelengths, a fiber coil $100 \mathrm{~m}$ long was heated to $60^{\circ} \mathrm{C}$.

It was verified from our measurements that RA-OTDR intensity is strongly dependent of peak power input, and small variations in peak-peak power can provoke more significant changes in RA-OTDR signal than wavelength variation, in order to solve this problem a more refined power control need to be adopted. Nevertheless it was realized at $70 \mathrm{~mW}$ average input power, the $1490 \mathrm{~nm}$ signal is systematic more intense than the others, it is believed that emission of EDFA amplifier contribute at this range, since it emits in a wide band.

\section{Conclusion}

We have presented experimental results from the spatial evolution of the stimulated Raman Stokes and antiStokes scattering in a standard optical fiber. By using an OTDR dedicated to each Raman branch it was verified that antiStokes band experiences an attenuation, as it was expected from the Raman susceptibility nature, besides the point where attenuation is minimum decay exponentially at the same rate that the point where Stokes is amplified. The susceptibility wavelength dependence was tested for three wavelengths belonging to the anti-Stokes band. The system was employed as a distributed temperature sensor in order to quantify the anti-Stokes signal deterioration as a function of power input. It was verified RA-OTDR signal for a heated portion of fiber can be completely deteriorated, which implies in a power input dependence of dynamical range and temperature sensibility for distributed Raman sensors in an opposite sense of spontaneous scattering, since in the present case one needs to decrease power input to go further in the optical fiber link. Finally, measurements for different anti-Stokes wavelength show no appreciable changes in a RA-OTDR amplitude signal from a heated portion of fiber in distributed sensor link.

\section{References}

[1] Dakin, J.P., Pratt, D.J., Bibby, G.W., Ross, J.N., “Temperature Distribution using Raman Ratio Thermometry”, Proc. SPIE 566, 249-256 (1985).

[2] Hartog, A.H., Leach, A.P., Gold, M.P. “Distributed Temperature Sensing in Solid-Core Fibres”, Elec. Lett., 21(23), 1061-1062 (1985).

[3] Park, J., et. al., "Raman-Based Distributed Temperature Sensor with Simplex Coding and Link Optimization", Photon. Techn. Lett. 18(17) 1879-1881 (2006).

[4] Signorini, A., Faralli, S., Soto, M., et. al., "40 km Long-Range Raman Based Distributed Temperature Sensor with Meter-Scale Spatial Resolution", Proc. OSA/OFC/NFOEC (2010).

[5] Boyd, R. [Nonlinear Optics], Academic Press, Burlington \& California, Chapter 10 (2008).

[6] Coen, S., Wardle, D.A., Harvey, J.D., "Observation of non-Phase-Matched Parametric Amplification in Resonant Nonlinear Optics”, Phys. Rev. Lett. 89(27), 273901-1 - 273901-4 (2002).

[7] Newbury, N.R., " Raman Gain: Pump-Wavelength Dependence in Single-Mode Fiber”, Opt. Lett., 27(14), 1232-1234 (2002). 